\title{
Dissection of the Barley 2L1.0 Region Carrying the 'Laevigatum' Quantitative Resistance Gene to Leaf Rust Using Near-Isogenic Lines (NIL) and subNIL
}

\author{
Thierry C. Marcel, ${ }^{1}$ Reza Aghnoum, ${ }^{1}$ Jérôme Durand, ${ }^{2}$ Rajeev K. Varshney, ${ }^{3}$ and Rients E. Niks ${ }^{1}$ \\ ${ }^{1}$ Laboratory of Plant Breeding, Graduate School for Experimental Plant Sciences, Wageningen University, \\ Droevendaalsesteeg 1, 6708 PB Wageningen, The Netherlands; ${ }^{2}$ I.U.P.-P.V.I.A., Faculté des Sciences, 33 rue Saint-Leu, \\ 80039 Amiens Cedex 1, France; ${ }^{3}$ Leibniz-Institute of Plant Genetics \& Crop Plant Research (IPK), Corrensstraße 3, \\ D-06466 Gatersleben, Germany
}

Submitted 20 March 2007. Accepted 9 July 2007.

Partial resistance to leaf rust (Puccinia hordei G. H. Otth) in barley is a quantitative resistance that is not based on hypersensitivity. This resistance hampers haustorium formation, resulting in a long latency period in greenhouse tests. The three most consistent quantitative trait loci (QTL) uncovered in the L94 × 'Vada' mapping population were introgressed by marker-assisted backcrossing into the susceptible L94 background to obtain near-isogenic lines (NIL). We also developed the reciprocal VadaNIL for the susceptibility alleles of those QTL. The QTL $R p h q 2$ affected latency period of $P$. hordei more than the QTL Rphq3 and Rphq4. The NIL confirmed the contribution of $R p h q 2$ to partial resistance by prolonging the latency period by $28 \mathrm{~h}$ on L94-Rphq2 and shortening the latency period by $23 \mathrm{~h}$ on Vada-rphq2. On the basis of flanking restriction fragment length polymorphism-based markers, $R p h q 2$ appeared to be located near the telomeric end of the long arm of chromosome $2 \mathrm{H}$, in a physical region of high recombination, making it the target QTL for map-based cloning. Microscopic observations on the NIL confirmed the nonhypersensitive nature of the resistance conferred by Rphq2. A high-resolution genetic map of the $R p h q 2$ region was constructed using a population of 38 subNIL with overlapping L94 introgressions in Vada background across the region. Rphq2 mapped approximately 2 centimorgans $(\mathrm{cM})$ proximal from the MlLa locus. By bulked segregant analysis and use of synteny with rice, we developed additional markers and fine-mapped $R p h q 2$ to a genetic interval of $0.11 \mathrm{cM}$ that corresponds to a stretch of sequence of, at most, $70 \mathrm{~kb}$ in rice. Analysis of this rice sequence revealed predicted genes encoding two proteins with unknown function, retrotransposon proteins, peroxidase proteins, and a protein similar to a mitogen-activated protein kinase kinase kinase (MAP3K). Possible homologs of those peroxidases and MAP3K in barley are candidates for the gene that contributes to partial resistance to $P$. hordei.

Corresponding author: R. E. Niks; E-mail: rients.niks@wur.nl

Current address of R. K. Varshney: International Crops Research Institute for the Semi-Arid Tropics (ICRISAT), Patancheru 502324 AP, India.

* The $\boldsymbol{e}$-Xtra logo stands for "electronic extra" and indicates that a supplemental table is published online. Figures 1, 2, 3, 4, 6, and 7 also appear in color online.
Additional keywords: Blumeria graminis f. sp. hordei, comparative mapping, Hordeum vulgare L., substitution mapping.

Genetic dissection of a character of interest is an essential step toward the map-based cloning of the gene or genes underlying this character. Map-based cloning, also called positional cloning, is the process of identifying the genetic basis of a mutant phenotype by looking for linkage to markers whose physical location in the genome is known (Jander et al. 2002). Although map-based cloning is still considered to be time-consuming and laborious, the availability of the whole genome sequences of Arabidopsis (Jander et al. 2002) and rice $(\mathrm{Xu}$ et al. 2005) greatly facilitates its process. For example, the occurrence of extensive synteny among cereal crops allows the use of the rice genomic sequence for comparative genome analysis (Devos 2005). Map-based cloning has been used to isolate a large number of genes that inherit according to Mendelian ratios; however, it has been considered problematic for quantitative trait loci (QTL) because genotypes cannot be unambiguously recognized from phenotypes of individual plants (Remington et al. 2001). A QTL gene is characterized by its quantitative effect, is dependent on the genetic background and on its allelic form, and requires QTL-mapping software to establish its position (Niks et al. 2004). The development of QTL near-isogenic lines (NIL) allows the evaluation of a QTL in a nearly uniform genetic background, overcoming the difficulties of identifying QTL phenotypes. In a QTL-NIL, the target QTL becomes the major genetic source of variation because of the absence of other segregating QTL. The QTL is considered Mendelized (Alonso-Blanco and Koornneef 2000). Then, by developing multiple subNIL with overlapping introgressions across the target region, substitution mapping can effectively dissect the QTL (Paterson et al. 1990). Indeed, most successes in cloning plant QTL have been obtained by following this approach (Salvi and Tuberosa 2005).

At Wageningen University, the partial resistance of barley (Hordeum vulgare L.) to leaf rust (Puccinia hordei G. H. Otth) has been studied since 1971. The measurement of latency period of the rust fungus has been shown to be the most reliable and effective method to quantify levels of partial resistance in a greenhouse test (Neervoort and Parlevliet 1978). A population of $103 \mathrm{~F}_{9}$ recombinant inbred lines (RILs) was developed from a cross between L94 and 'Vada' (Qi et al. 1998a). L94 is a line from an Ethiopian landrace extremely susceptible to bar- 
ley leaf rust. Vada is an obsolete Dutch cultivar developed from the cross ' $H$. laevigatum' $\times$ 'Gold' (Dros 1957), which has a high level of partial resistance to $P$. hordei (Neervoort and Parlevliet 1978; Niks 1982). QTL mapping in L94 $\times$ Vada identified six QTL (Qi et al. 1998b). The three QTL that showed the largest and most consistent effect (i.e., Rphq2, $R p h q 3$, and $R p h q 4$ ) were introgressed into the susceptible L94 background by marker-assisted backcrossing to obtain NIL (Van Berloo et al. 2001). Rphq2 is located on chromosome $2 \mathrm{HL}$ in the 2L1.0 region described by Dilbirligi and associates (2005). The $2 \mathrm{~L} 1.0$ region is one of the largest gene-rich regions in wheat and barley and is highly syntenic between those two cereals. The $R p h q 2$ locus, together with the powdery mildew (Blumeria graminis f. sp. hordei) resistance gene MlLa (Giese et al. 1993) and the barley leaf stripe (Pyrenophora graminea) resistance gene Rdgla (Arru et al. 2002; Thomsen et al. 1997), has been transferred to European barley cultivars from the 'botanical' barley line ' $H$. laevigatum' (Jensen and Jørgensen 1992). This obscure donor accession does not belong to a valid species, but should be considered a regular $H$. vulgare accession. Rphq3 is located near the centromere of chromosome $6 \mathrm{H}$ and Rphq4 is on the satellite chromosome 5HS.

The objective of this study was to characterize macroscopically, microscopically, and molecularly the three most consistent QTL identified in order to select the best candidate for cloning a gene for partial resistance to barley leaf rust. Information on the size of the effect of each QTL in its NIL background and on recombination frequencies in the three QTL regions are the basis on which to make this selection. The genetic region of $R p h q 2$ was saturated with molecular markers available in the literature, and identified by following a bulk segregant analysis or by making use of the synteny between rice and barley. Finally, the gene for quantitative resistance to leaf rust Rphq2 and the powdery mildew resistance gene $M l L a$ were finemapped by substitution mapping.

\section{RESULTS}

Construction of QTL-NIL

containing $\mathrm{L94}$ and Vada introgressions.

Two sets of NIL were generated in the genetic backgrounds of L94 and Vada (Fig. 1). Van Berloo and associates (2001) developed NIL containing the QTL Rphq2, Rphq3, and Rphq4 in the susceptible background of L94. Genotyping with 226 new amplified fragment length polymorphism (AFLP) markers resulted in an average distance between adjacent markers of 4.1 centimorgans (cM) and revealed one unwanted Vada introgression at the telomeric end of chromosome $3 \mathrm{H}$ in L94-Rphq3 and two unwanted Vada introgressions on chromosomes $3 \mathrm{H}$ and $6 \mathrm{H}$ in L94-Rphq4. L94-Rphq4 was backcrossed (BC) a fourth time to the recurrent parent L94 and a new NIL was selected that did not contain the two unwanted Vada introgressions. A NIL combining the two QTL $R p h q 2$ and $R p h q 3$ was selected successfully with microsatellite markers in a segregating $\mathrm{F}_{2}$ progeny derived from a cross between L94-Rphq2 and L94-Rphq3 (Fig. 1).

Reciprocal Vada NIL were generated by selecting for a single donor genome introgression carrying $R p h q 2$ or $R p h q 6$ at the $\mathrm{BC}_{4}$ generation and Rphq3 or Rphq4 at the $\mathrm{BC}_{5}$ generation. The 157 AFLP markers used to select the Vada NIL did not reveal any unwanted L94 fragment. The average distance between two adjacent AFLP markers was $5.6 \mathrm{cM}$. Plants containing interesting recombination events for fine-mapping the QTL $R p h q 2$ and Rphq3 were identified and retained during the selection process (i.e., Vada-rph $q 2^{b}$, Vada-rph $q 2^{c}$, and Vada$\left.r p h q 3^{b}\right)$. As counterpart to the L94 NIL, a Vada NIL combining the QTL Rphq2 and Rphq3 also was selected in the $\mathrm{BC}_{5}$ gen- eration. All $\mathrm{BC}_{4}$ - and $\mathrm{BC}_{5}$-selected plants were selfed to select for NIL with homozygous introgressions.

\section{On seedlings, $R p h q 2$ prolongs the latency period of Puccinia hordei more than Rphq3.}

The effect of a QTL may depend on its genetic background and different QTL may have different size of effect. Therefore, it is necessary to evaluate the phenotypes of the NIL before embarking on map-based cloning experiments. A greenhouse disease test on seedlings is a fast and reliable method for the phenotypic evaluation of the QTL-NIL in repeated experiments. In the RIL population, Qi and associates (1998b) identified Rphq2 and Rphq3 as being the two QTL with the greatest effect in the seedling stage, whereas they identified $R p h q 4$ and Rphq6 only in the adult plant stage and in the field. The evaluation of the NIL confirmed that Rphq2 had a significant effect in seedlings (Table 1; Fig. 2). Rphq2 prolonged the latency period (LP) by $28 \mathrm{~h}$ on L94-Rphq2 whereas the alternative $r p h q 2$ allele shortened the LP by $23 \mathrm{~h}$ on Vada-rphq2. In the NIL, Rphq3 did not have a significant effect in seedlings compared with the parental lines L94 and Vada (Table 1). Nevertheless, Rphq3 prolonged the LP by $9 \mathrm{~h}$ on L94-Rphq3 whereas the alternative allele shortened the LP by $7 \mathrm{~h}$ on Vadarphq3. The combination of Rphq2 and Rphq3 in the same NIL background resulted in levels of resistance higher than those of any NIL with a single QTL introgression (Table 1). However, these differences were not always statistically significant. In L94 and in Vada background, both introgressed Rphq2 and $R p h q 3$ alleles, or $r p h q 2$ and $r p h q 3$ alleles, even resulted in a relative latency period (RLP) similar to that on Vada and L94,

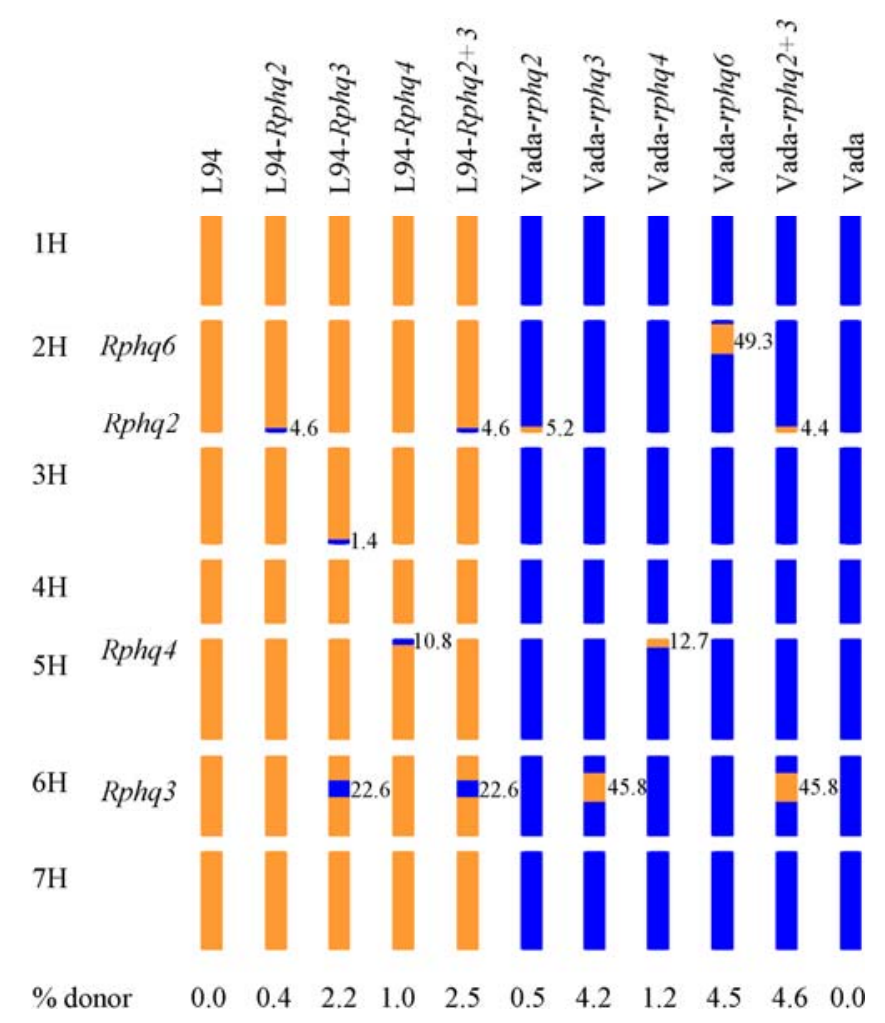

Fig. 1. Graphical genotypes of four near-isogenic lines (NIL) carrying the Vada allele of quantitative trait loci (QTL) or a combination of QTL in the susceptible L94 genetic background and of five reciprocal NIL carrying the L94 allele of QTL or a combination of QTL in the partially resistant Vada genetic background. The name of a QTL is indicated at its approximate position on the corresponding linkage group. The size of the introgressions (in centimorgans) are indicated on the right side of the chromosome bars. Percent donor is the estimated percentage of donor-derived genome remaining in the corresponding NIL. 
respectively. It indicates that these two QTL explain nearly all the genotypic variation observed at seedling stage. As expected on the basis of the results of Qi and associates (1998b), the LP of the rust on seedlings of L94-Rphq4, Vada-rphq4, and Vadarphq6 was not significantly different from that on L94 and Vada, respectively (Table 1).

\section{Microscopic observations confirm the prehaustorial type of resistance conferred by $R p h q 2$.}

The ranking of the genotypes on the basis of microscopically assessed percentages of early aborted colonies and sizes of established colonies was similar to ranking on the basis of the macroscopically assessed levels of partial resistance (Fig. 3). Vada and Vada-rphq3 caused the highest RLP (Fig. 3A), and had a high percentage of early aborted colonies (Fig. 3B) and a short diameter of established colonies (Fig. 3C). A high degree of early abortion without plant cell necrosis is indicative of the failure of haustorium formation by the infection unit, which results in low levels of infectibility by Puccinia hordei as observed on Vada (Niks 1982, 1983, 1986). L94Rphq2 and L94-Rphq2+3 had significant macroscopic and microscopic effects on the level of partial resistance (Fig. 3). The percentage of early aborted colonies in L94-Rphq2 was not significantly different from the one in L94 in this experiment but was, in quantity, three times higher than in L94, L94$R p h q 3$, or Vada-rphq2+3 (Fig. 3B). Approximately $69 \%$ of the early aborted colonies in L94-Rphq2 were not associated with host cell necrosis, confirming the predominantly prehaustorial type of resistance conferred by this gene. The total proportion of colonies (early aborted and established) associated with host cell necrosis ranged from $16 \%$ for Vada-rphq2 to $53 \%$ for Vada-rphq2+3. However, the necrosis occurred frequently as only one cell in relatively large established colonies, and was not particularly strong in Vada or NIL carrying Rphq2 and Rphq3. This suggests that the necrosis was not a relevant factor explaining the level of partial resistance conferred by the QTL. The three lines carrying the L94 allele of Rphq2 consistently had a lower RLP (Fig. 3A), a lower percentage of early

Table 1. Relative latency period at seedling stage of Puccinia hordei isolate 1.2.1 on barley recombinant inbred lines (RIL) and near-isogenic lines (NIL) that differ for $R p h q$ genes

\begin{tabular}{lccllll}
\hline & \multicolumn{3}{c}{ RIL $^{\mathbf{v}}$} & & \multicolumn{2}{c}{ NIL } \\
\cline { 2 - 3 } \cline { 7 - 8 } Gene & Fitted value $^{\mathbf{w}}$ & Mean $^{\mathbf{x}}$ & SE $^{\mathbf{y}}$ & & Mean $^{\mathbf{z}}$ & SE \\
\hline L94-Rphq2 & 110 & $109 \mathrm{~b}$ & 2.17 & & $116 \mathrm{bc}$ & 1.80 \\
L94-Rphq3 & 108 & $109 \mathrm{~b}$ & 1.10 & & $106 \mathrm{ab}$ & 1.80 \\
L94-Rphq4 & 102 & $103 \mathrm{ab}$ & 1.93 & & $100 \mathrm{a}$ & 2.07 \\
L94-Rphq2+3 & 117 & $119 \mathrm{c}$ & 0.91 & & $120 \mathrm{bc}$ & 1.77 \\
L94 & $\ldots$ & $101 \mathrm{a}$ & 1.44 & & $100 \mathrm{a}$ & 1.77 \\
Vada-rphq2 & 113 & $110 \mathrm{~b}$ & 1.23 & & $115 \mathrm{abc}$ & 1.83 \\
Vada-rphq3 & 115 & $109 \mathrm{~b}$ & 1.68 & & $125 \mathrm{c}$ & 2.12 \\
Vada-rphq4 & 121 & $124 \mathrm{c}$ & 1.93 & & $121 \mathrm{c}$ & 1.87 \\
Vada-rphq6 & 122 & $124 \mathrm{c}$ & 1.88 & & $128 \mathrm{c}$ & 2.19 \\
Vada-rphq2+3 & 106 & $106 \mathrm{ab}$ & 1.83 & & $106 \mathrm{ab}$ & 2.54 \\
Vada & $\ldots$ & $124 \mathrm{c}$ & 1.51 & & $127 \mathrm{c}$ & 1.80 \\
\hline
\end{tabular}

${ }^{v}$ According to Qi and associates (1998b).

${ }^{\text {w }}$ Theoretical value calculated based on the RIL population mean and the allelic effect of each quantitative trait locus (QTL) as determined by MapQTL 5.0.

${ }^{x}$ Mean value of the RIL carrying the QTL allele considered and the QTL allele of the recurrent parent at the other QTL predicted from regression model; means followed by a common letter are not significantly different according to Duncan's test $(P<0.05)$.

y $\mathrm{SE}=$ standard error estimates.

${ }^{\mathrm{z}}$ Mean value of the considered NIL predicted from regression model (100\% L94 corresponds with an approximately 171-h latency period); means followed by a common letter are not significantly different according to Duncan's test $(P<0.05)$. aborted colonies (Fig. 3B), and a longer diameter of established colonies (Fig. 3C) than the three lines carrying the Vada allele of Rphq2.

\section{Selection of $R p h q 2$ as the target QTL to clone a gene for partial resistance.}

Nine primer pairs designed on barley restriction fragment length polymorphism (RFLP) sequences were converted into locus-specific polymerase chain reaction (PCR) markers and mapped in the L94 × Vada population (Marcel et al. 2007). Three of the mapped primer pairs gave direct sequence-characterized amplified region (SCAR) markers (ABG458A, MWG618, and MWG835), while the other six gave cleaved amplified polymorphic sequence (CAPS) markers (Prx2_AciI, MWG2200_AvaI, MWG2068_HaeIII, cMWG679_BssKI, ABG388_NlaIII, and MWG502_HpaII). With the exception of cMWG679, all markers mapped in the vicinity of the target QTL. The CAPS marker cMWG679 mapped at the telomeric end of chromosome $6 \mathrm{HS}$ in L94 $\times$ Vada (data not shown) whereas the corresponding RFLP marker had been mapped 60 $\mathrm{cM}$ proximal on that same chromosome arm, near the position of Rphq3 (Graner et al. 1991). MWG2200 and MWG2068 flanked Rphq2 on chromosome 2HL (Fig. 4) and delimit a physical region of high recombination of 1.1 to $1.8 \mathrm{Mb} / \mathrm{cM}$ (Künzel et al. 2000; Stephens et al. 2004). ABG458A and ABG388 flanked $R p h q 3$ and mapped on both sides of the centromeric region of chromosome $6 \mathrm{H}$ (Fig. 4). They delimit a physical region of suppressed recombination of 28.8 to $42.0 \mathrm{Mb} / \mathrm{cM}$ (Künzel et al. 2000; Stephens et al. 2004). MWG502 and MWG835 are closely linked to Rphq4 on chromosome 5HS (Fig. 4) and delimit a physical region of very high recombination, with less than $0.9 \mathrm{Mb} / \mathrm{cM}$ (Künzel et al. 2000; Stephens et al. 2004).

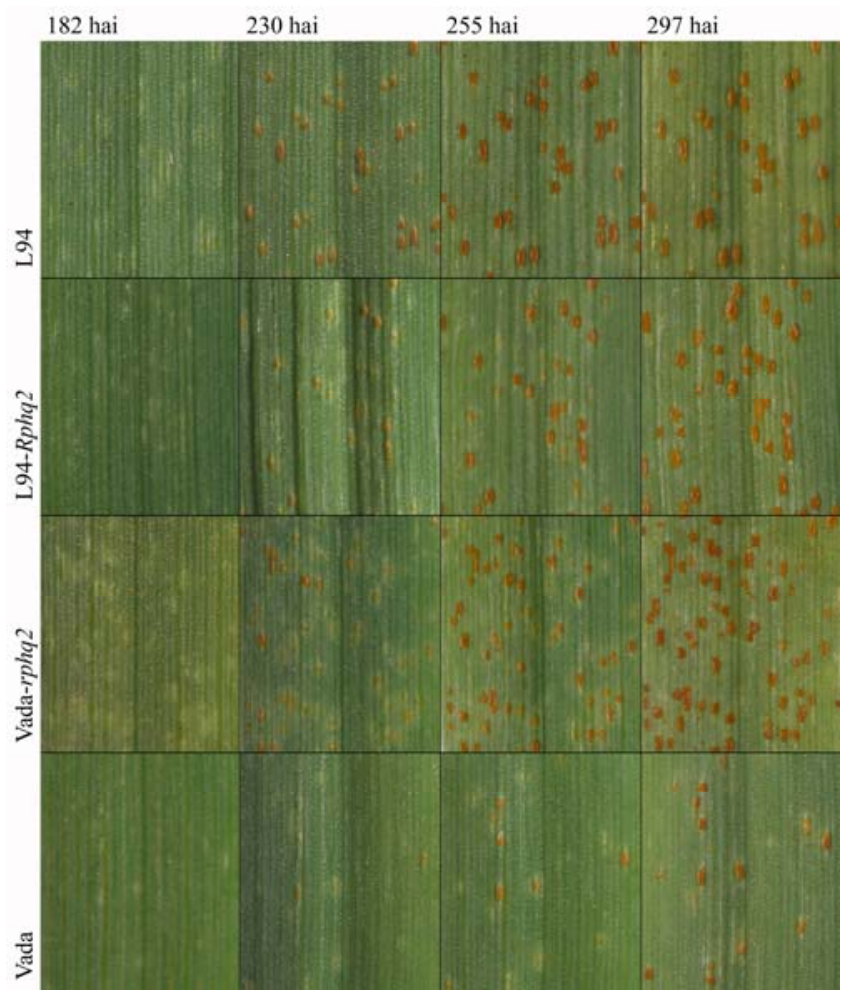

Fig. 2. First leaf of barley seedlings from the susceptible line L94, the nearisogenic lines (NIL) L94-Rphq2 and Vada-rphq2, and the partially resistant line Vada at 182, 230, 255, and 297 h after inoculation (hai) with Puccinia hordei 1.2.1. For each line, the same leaf area is shown at four different time points. The latency period on the corresponding seedlings were 190, 208, 205, and $237 \mathrm{~h}$, respectively. 
Rphq2 is the easiest QTL to detect in seedlings of the corresponding L94 NIL and Vada NIL (Table 1; Fig. 2). Microscopic observations confirmed the prehaustorial type of resistance conferred by Rphq2 (Fig. 3). On the basis of flanking RFLPbased markers, $R p h q 2$ also appeared to be located in a physical region of high recombination, making it the QTL of choice for map-based cloning.

\section{Marker saturation of the region containing $R p h q 2$}

by bulked segregant analysis and synteny-based approaches.

To efficiently identify molecular markers linked to Rphq2, on the distal end of chromosome 2HL, a bulked segregant analysis (BSA) was performed by using AFLP on resistant and susceptible pools of L94 $\times$ Vada RILs and on the NIL L94Rphq2. By using a total of 48 Pst I/MseI primer combinations, eight AFLP markers were identified that were present only in the resistant pool, in Vada, and in L94-Rphq2, and five AFLP markers were identified that were present only in the susceptible pool and in L94. All 13 AFLP markers mapped in the L94 $\times$ Vada RIL population within the 4.6-cM Vada genomic segment introgressed into L94-Rphq2. Two previously mapped EcoRI/MseI AFLP, E40M32-402 and E42M48-376, and one new PstI/MseI AFLP, P15M53-435, were successfully converted into one CAPS and two SCAR markers designated caE40M32402, scE42M48-376, and scP15M53-435, respectively. In L94 $\times$ Vada, caE40M32-402, scE42M48-376, and scP15M53-435 mapped at the expected position of the corresponding AFLP markers (Fig. 5A).

We further saturated the region of $R p h q 2$ with molecular markers by exploiting the synteny between rice and barley. The alignment of the genomes of major grass species indicated a syntenic relationship between the triticeae chromosome 2 and the rice chromosomes 4 and 7 (Devos 2005; Moore et al. 1995). The blastn analysis of barley expressed sequence tags (EST) identified a region on rice chromosome 4 as being syntenic with the region of $R p h q 2$. The rice target region was covered by a contig of four bacterial artificial chromosome (BAC) clones spanning a physical distance of $562 \mathrm{~kb}$ : OSJNBa0088H09, OSJNBa0070M12, OSJNBa0039K24, and OSJNBb$00220 J 19$ (Fig. 5C). We designed 19 primer pairs based on the sequences of 19 barley EST with highest homology to 19 predicted rice genes $\left(3.10^{-19}<\right.$ Expect $\left.<3.10^{-238}\right)$ from the four rice BACs. Of the 19 primer pairs designed, eight were converted into locus-specific PCR markers and mapped in the L94 $\times$ Vada population. Seven markers (WBE001, WBE110, WBE111, and WBE113 to WBE116) mapped on barley chromosome 2HS within the L94 genomic segment introgressed in Vada-rphq2 (Fig. 5B and C), while the eighth (WBE112: Expect $=3.10^{-51}$ ) mapped on chromosome 3HS. Those EST-based markers were designated WBEnr for Wageningen Barley EST. WBE110 was the best barley homolog to two predicted rice genes on OSJN$\mathrm{Ba} 0088 \mathrm{H} 09$ that encode exocyst subunit Exo70 proteins, and WBE114 was the best barley homolog to three predicted rice genes on OSJNBa0039K24 that encode peroxidase proteins (Supplementary Table S1). Except for an inversion between WBE110 and WBE113, the order of the WBEnr markers on the barley high-resolution map (Fig. 5B) was in agreement with the order of the predicted genes on rice chromosome 4 .

Altogether, the employed strategies saturated the region of Rphq2 with an average density of one marker per $0.15 \mathrm{cM}$ (Fig. 5A). In all, 35 markers mapped within the 5.2-cM L94 genomic segment introgressed in Vada-rphq2 (Fig. 6): seven EcoRI/MseI AFLPs identified by Qi and associates (1998a), 13 Pst $\mathrm{I} / M s e$ I AFLPs identified by BSA (this study), two RFLP converted into locus-specific PCR markers (this study), seven EST-based markers developed by synteny with rice (this study), MWG097 obtained from Mohler and Jahoor (1996), k00345
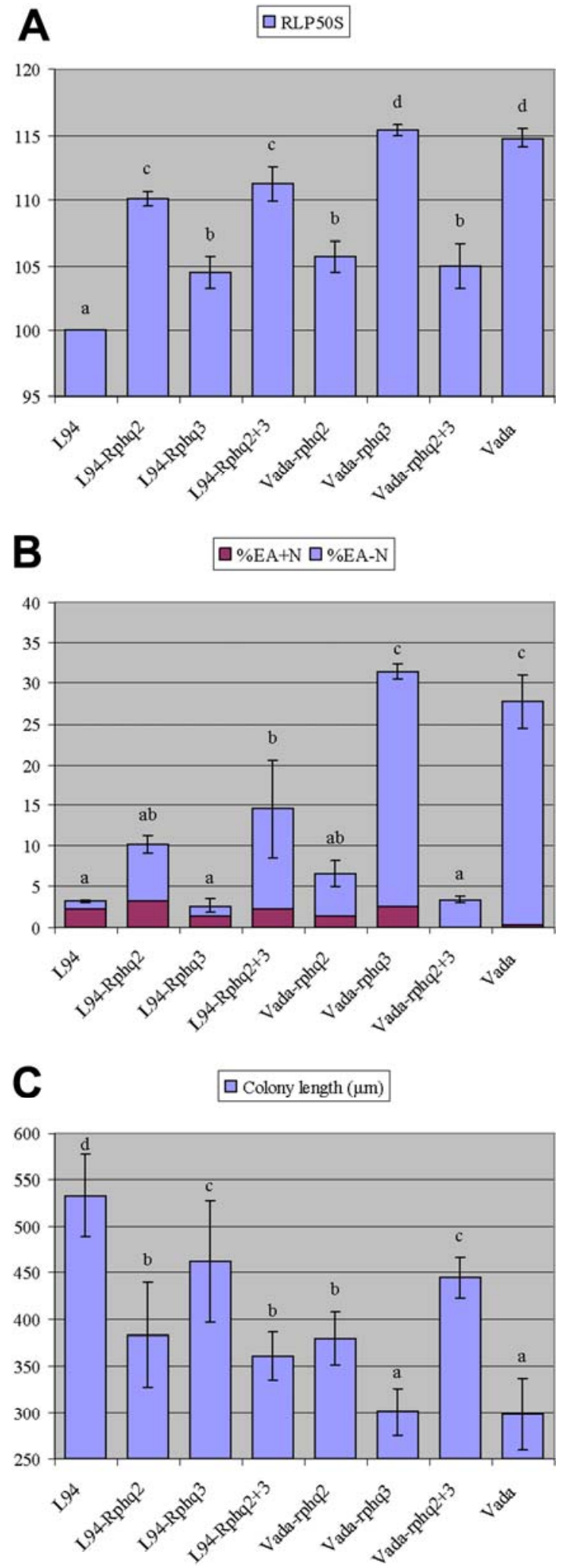

Fig. 3. Macroscopic and microscopic effects of quantitative trait loci (QTL) near-isogenic lines (NIL) on the level of partial resistance to barley leaf rust. Histograms of $\mathbf{A}$, the relative latency period at seedling stage (RLP50S), B, the proportion of early aborted colonies associated (\% $\mathrm{EA}+\mathrm{N})$ or not $(\%$ EA-N) with host cell necrosis, and $\mathbf{C}$, the length of established colonies in micrometers after infection with Puccinia hordei isolate 1.2.1 on L94, Vada, and QTL NIL. For microscopic observations, leaf segments were sampled $110 \mathrm{~h}$ after inoculation. Error bars, \pm standard error; similar letters on bars indicate that the means do not differ significantly according to Duncan's test $(P<0.05)$. 
obtained from Hori and associates (2005) and four simple sequence repeats obtained from Varshney and associates (2007). Only the 27 most distal markers mapped within the Vada genomic segment introgressed in L94-Rphq2 (Fig. 5A).

\section{Substitution mapping places $R p h q 2$}

\section{to a genetic interval of $0.11 \mathrm{cM}$.}

The genetic fine-mapping of a target locus is an essential step preceding the map-based isolation of a gene. We screened $923 \mathrm{BC}_{4} \mathrm{~S}_{2}$ seedlings (i.e., 1,846 gametes) segregating for the L94 fragments introgressed in Vada-rphq 2 or in Vada-rph $q 2^{b}$ with the microsatellites GBM1475 and GBMS216. A set of 38 seedlings was identified that recombined between these two markers, implying a $2.1 \%$ recombination rate (Fig. $5 \mathrm{~B})$. This is two times less than the original 4.6-cM genetic interval calculated between those markers in the L94 $\times$ Vada map (Fig. 5A). One of the 38 seedlings had a double recombination that resulted in the subNIL v040102. The disease test on the subNIL resolved $R p h q 2$ in a single, incompletely dominant gene cosegregating with the AFLP markers P15M51-204, P14M50-225, and P14M54-252 (Fig. 6). SubNIL with an RLP value statistically significantly lower than the RLP on Vada were considered to carry the L94 allele of Rphq2, whereas subNIL with an RLP value not statistically different from the RLP on Vada were considered to carry the Vada allele of Rphq2. The two resulting groups of subNIL, distinguished according to their phenotype, did not show any discrepancy with the corresponding genotypes (Fig. 6). The minimum difference between RLP50S values of two subNIL carrying different alleles of Rphq2 was equal to 6.9 (i.e., v031002 and v050304) corresponding to a prolongation of the LP of the leaf rust fungus of approximately $13 \mathrm{~h}$, whereas the average difference between subNIL carrying different alleles of $R p h q 2$ was equal to 14, corresponding to a prolongation of approximately $26 \mathrm{~h}$. QTL mapping with the phenotypic and genotypic data of the subNIL population also indicated P15M51-204, P14M50-225, and P14M54-252 as peak markers for Rphq2 (Fig. 6). Two EST-based markers, WBE114 and WBE115, flanked these three AFLPs in a genetic interval of approximately $0.11 \mathrm{cM}$ that corresponds to a rice syntenic stretch of sequence of 31.4 to $69.7 \mathrm{kbp}$. The incertitude is due to WBE114 being the best blastn hit for three different predicted rice genes encoding peroxidase proteins on the BAC OSJNBa0039K24. The marker order in the subNIL population agrees with the one on the L94 $\times$ Vada map (Fig. $5 \mathrm{~A}$ and $\mathrm{B}$ ) and no inconsistency was found between the phenotype and the genotype of the 38 subNIL (Fig. 6).

The powdery mildew resistance gene $M l L a$ also segregated in the 38 subNIL. On the basis of the infection types (Fig. 7), MlLa was mapped distal to the microsatellite GBMS216 within the 1.2-cM chromosome segment differing between Vada-rphq2 and Vada-rphq $2^{b}$ (Figs. 5A and 6). Additional marker analyses explained the infection type (IT) of $0.0 \mathrm{ob}-$ served on v010101 by the presence of a remaining L94 fragment carrying the mlo gene that segregated in the $\mathrm{BC}_{4} \mathrm{~S}_{1}$ generation of Vada-rphq $2^{b}$ (data not shown). The genetic distance between the two AFLP markers flanking mlo, among the 157 AFLP markers used to select the Vada NIL, was $28 \mathrm{cM}$. This is one of the biggest gaps observed between two adjacent AFLP markers used to select the Vada NIL. The NIL and 37 other subNIL did not have this unwanted L94 fragment on chromosome $4 \mathrm{HL}$.

\section{DISCUSSION}

\section{Verification of QTL \\ for partial resistance to $P$. hordei in NIL of barley.}

Recent technical advancements and refinements of analytical methods have enabled the molecular dissection of loci responsible for the genetic control of quantitative traits (Salvi and Tuberosa 2005). Nevertheless, the reliability at which a QTL effect can be assessed is the cornerstone toward the mapbased isolation of the underlying genes. Out of the six QTL for partial resistance to barley leaf rust identified by Qi and associates (1998b), the three loci explaining most of the variation (i.e., more than $15 \%$ per QTL) have been introduced into NIL and reciprocal NIL (Fig. 1). Compared with the results obtained on the RIL population (Qi et al. 1998b), our data on NIL confirm the substantial effect of Rphq2 on LP of $P$. hordei and the lack of effect of Rphq4 in the seedling stage (Table 1). In the RIL population, the effect of Rphq2 had been assessed as $35 \%$ of the phenotypic variation whereas, in the NIL, this proportion appeared to be approximately $50 \%$. The consistent
$2 \mathrm{H}$

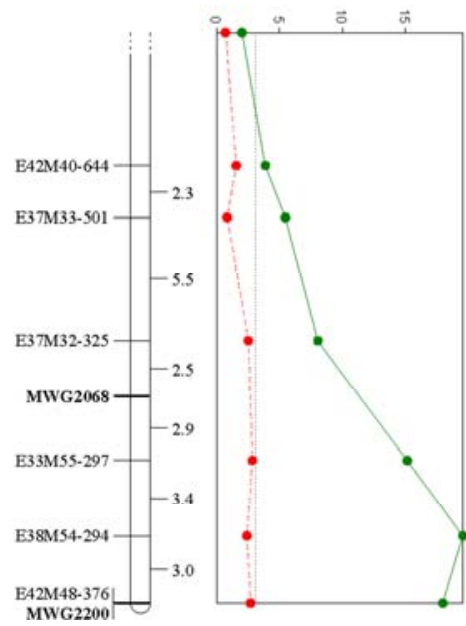

$5 \mathrm{H}$

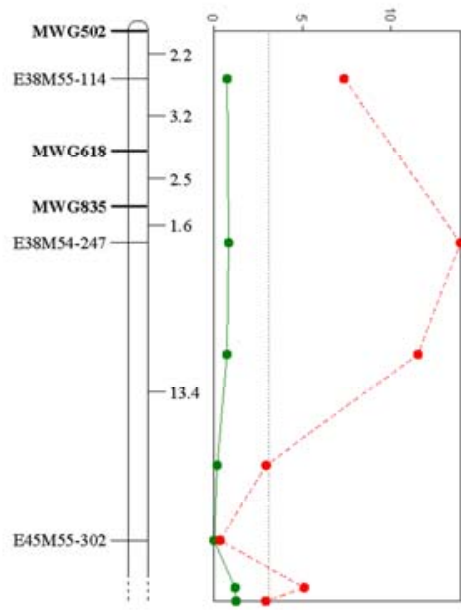

$6 \mathrm{H}$

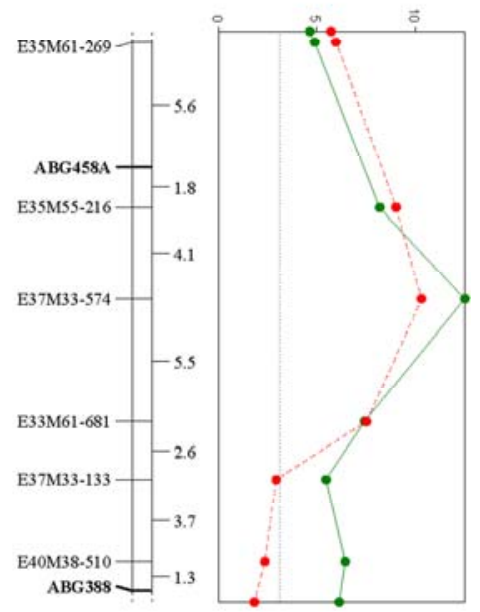

Fig. 4. Log of the likelihood ratio profiles of $R p h q 2, R p h q 3$, and $R p h q 4$ along the genetic distances of the L94 $\times$ Vada genetic linkage map at the seedling stage (green solid line) and at the adult plant stage (red dashed line), obtained by restricted-MQM mapping with the data of Qi and associates (1998b). Marker names are on the left side of the chromosomes and the genetic distances between two consecutive loci (in centimorgans) are indicated on the right side of the chromosomes. Markers in bold are locus-specific polymerase chain reaction markers converted from restriction fragment length polymorphism markers mapped on the physical maps of Künzel and associates (2000). 
and robust nature of Rphq2 in spring barley was confirmed by its appearance in a recent linkage disequilibrium (association) mapping study on Western-European spring barleys (Kraakman et al. 2006) and its detection in the mapping population derived from a cross between SusPtrit and Vada (Jafary et al. 2006). In the RILs, the phenotypic effect of Rphq3 was stronger than observed in NIL backgrounds (Table 1). Apparently, the size of the effect of Rphq3 depends on interaction with other genes, in particular Rphq2. Indeed, the RLP on Vada-rphq3 was similar to the RLP on Vada, indicating the absence of effect of $r p h q 3$ alone, whereas the RLP on Vada-rphq2+3 was lower than the RLP on Vada-rphq2 (Table 1), indicating a substantial effect of $r p h q 3$ in the presence of $r p h q 2$. This was confirmed by the proportion of early aborted colonies and the length of colonies microscopically measured on those NIL (Fig. 3). It is surprising that this interaction was not detected in the RIL population, in which Qi and associates (1998b) instead reported an interaction between Rphql and Rphq2.
Partial resistance is due to a prehaustorially acting mechanism and is not based on hypersensitivity (Niks 1982, 1983, 1986). However, the confirmation that individual genes for partial resistance involve the same mechanisms has never been demonstrated to our knowledge. The percentage of early aborted colonies on L94-Rphq2 was three times higher than the percentage of early aborted colonies on L94, whereas the percentage of early aborted colonies on L94-Rphq3 was similar to that on L94 (Fig. 3). Depending on the genotype, a varying proportion of colonies was associated with host cell necrosis. Nevertheless, the necrosis occurred frequently as only one cell in relatively large established colonies, and was not particularly strong in Vada or NIL carrying Rphq2 and Rphq3. The fact that $69 \%$ of the early aborted colonies on L94-Rphq2 were not associated with necrosis confirms that $R p h q 2$ confers a predominantly prehaustorial type of resistance to $P$. hordei.

In conclusion, only Rphq2 has a clear effect in the logistically convenient seedling stage and is an ideal candidate for

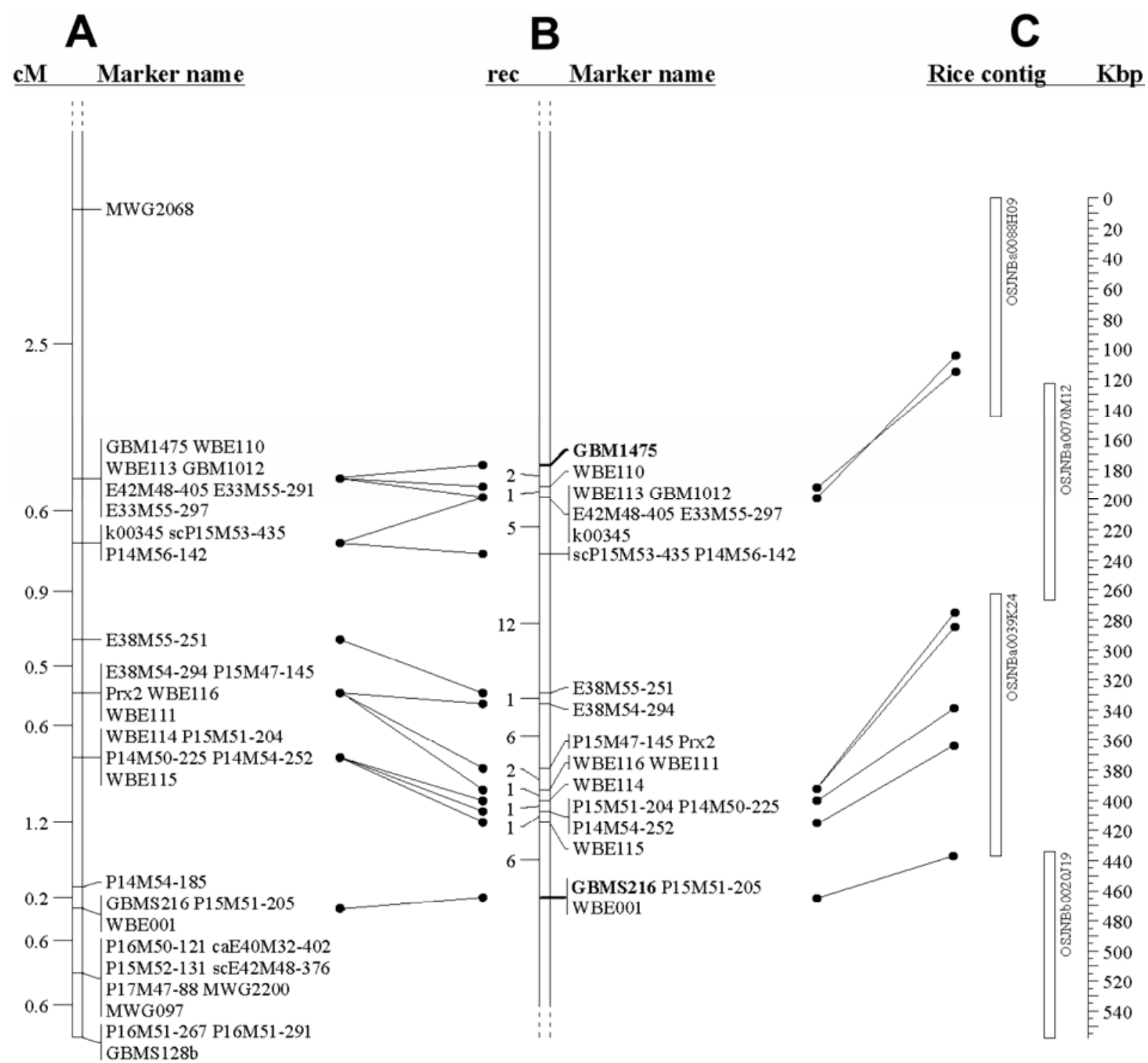

Fig. 5. Alignment of two genetic linkage maps covering the Rphq2 region on barley chromosome $2 \mathrm{HL}$ with the homologous region on rice chromosome 4 . A, The first map was constructed using a population of 103 recombinant inbred lines derived from the cross L94 × 'Vada'. B, Microsatellites GBM1475 and GBMS216 (in bold) were used to screen a population of 923 plants, and the resulting 38 sub-near-isogenic lines used to construct the second map. C, The seven markers derived from rice expressed sequence tags (WBE001, WBE110, WBE111, WBE113, WBE114, WBE115, and WBE116) allowed alignment to a rice physical contig of $557.5 \mathrm{~kb}$ comprising four bacterial artificial chromosome clones. Lines between $\mathrm{B}$ and $\mathrm{C}$ indicate the position of homologues present in the rice sequence. Genetic distances (in centimorgans $[\mathrm{cM}]$ ) observed for specific intervals are shown in A whereas the number of recombination identified between consecutive markers are shown in B. Physical scale (Kbp) in rice is indicated on the right in C. 


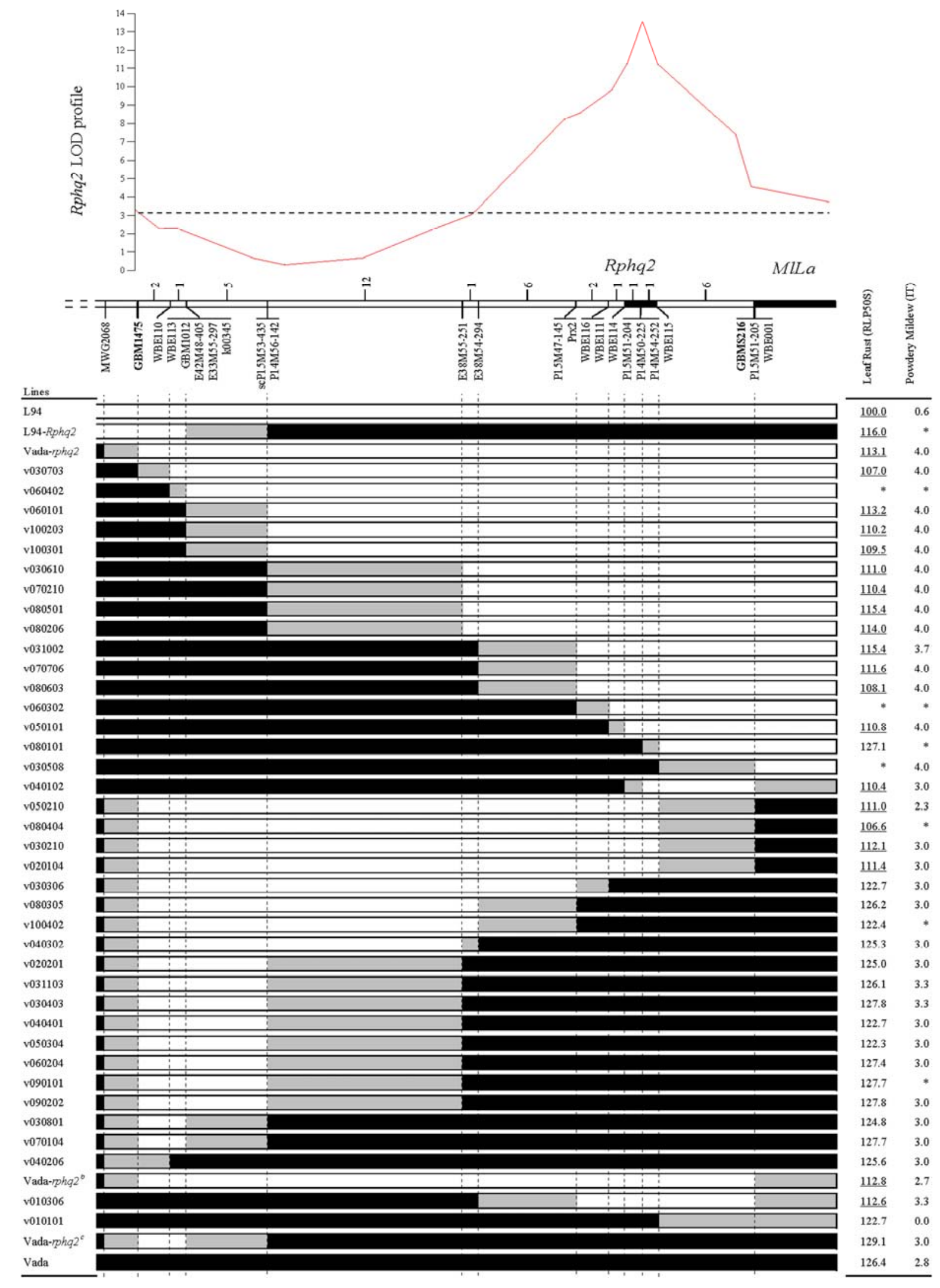

Fig. 6. Graphical genotypes, leaf rust (latency period, RLP50S) and powdery mildew (infection type, IT) phenotype means, and RLP50S log of the likelihood ratio profile for near-isogenic lines (NIL) and subNIL covering the L94 introgression on Vada-rphq2 NIL. Open bars represent homozygous L94, solid bars represent homozygous Vada, and gray bars represent intervals containing a recombination event. RLP50S values significantly different from the RLP50S value on the partially resistant line Vada are underlined $(4.43<$ least significant difference $[P<0.05]<8.38)$. Missing values are indicated by an asterisk (*). The number of recombinants identified between two consecutive markers is indicated within each marker interval on the chromosome bar. The markers in bold were used to identify recombination events within the L94 introgressions of Vada-rphq2 and Vada-rphq2 ${ }^{b}$. The putative positions of $R p h q 2$ and $M l L a$ are indicated by solid areas on the chromosome bar. 
map-based isolation experiments at this plant stage. However, the phenotypic effects of Rphq3 and Rphq4 in L94 genetic background were confirmed at the adult plant stage in repeated field trials (unpublished data), making these two QTL possible targets for map-based isolation experiments at the logistically less convenient adult plant stage.

\section{Construction of a high-resolution genetic map at the telomeric end of chromosome $2 \mathrm{HL}$.}

RFLP probes have been used to construct the earliest barley linkage maps (Graner et al. 1991; Kleinhofs et al. 1993), which then have been used to integrate different barley genetic maps (Karakousis et al. 2003; Langridge et al. 1995; Marcel et al. 2007; Qi et al. 1996) and to anchor barley physical maps (Künzel et al. 2000; Stephens et al. 2004). However, RFLP markers are becoming too labor intensive and time consuming to be used in large-scale experiments, and their conversion into locus-specific PCR markers greatly helped the introgression of QTL into the respective NIL. The converted RFLP markers also were decisive in estimating recombination frequencies within the QTL regions on the barley physical maps (Künzel et al. 2000; Stephens et al. 2004). On the basis of flanking RFLPbased markers, Rphq2 was localized in a physical region of high recombination (i.e., 1 to $2 \mathrm{Mb} / \mathrm{cM}$ ). Because $R p h q 2$ also was the easiest QTL to detect in seedlings (Table 1), it became our QTL of choice for map-based cloning. Two strategies were employed to further saturate the introgressions of L94-Rphq2 and Vada-rphq2 with molecular markers: a BSA approach and a synteny-based approach.

AFLP assays generate a high level of polymorphism and allow the simultaneous identification of a large number of amplification products. Those properties are suitable to identify
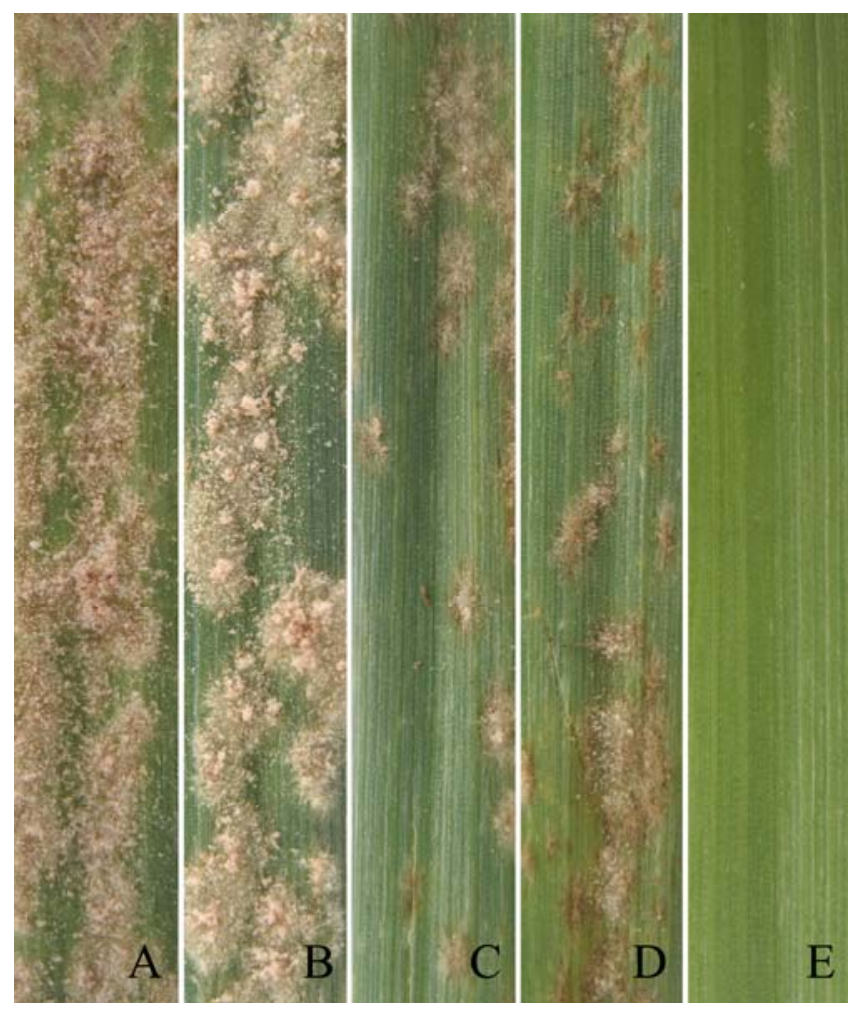

Fig. 7. First leaf of barley seedlings 12 days after inoculation with Blumeria graminis isolate C15. A, Susceptible control 'Manchuria' and B, near-isogenic line (NIL) Vada-rphq2 show an infection type (IT) of 4. C, Cv. Vada and D, SubNIL v40102 show an IT of 3, characteristic of the avirulence to MlLa. E, Line L94 shows an IT of 0, characteristic of the presence of $m l o$. molecular markers within a restricted region of the linkage map by BSA. Following this strategy, we successfully identified 13 AFLP markers within the 4.6-cM Vada segment introgressed into L94-Rphq2 after testing 48 PstI/MseI primer combinations. This number of markers identified is higher than expected on the basis of the number of markers found by Qi and associates (1998a) in the same DNA segment; they found four AFLP markers after running $25 \mathrm{EcoRI} / \mathrm{Mse}$ I primer combinations. Three of the AFLP markers identified by BSA cosegregated with Rphq2 (Fig. 6). Those markers are of utmost interest to identify BAC clones and to construct a physical map spanning Rphq2. The conversion of these markers into locusspecific PCR markers will facilitate this process.

A syntenic relationship between rice chromosome 4 and the long arm of barley chromosome $2 \mathrm{H}$ has been reported by several authors (Devos 2005; Moore et al. 1995; Schmierer et al. 2003). This syntenic relationship was confirmed in our study, in which seven barley EST identified by homology to predicted rice genes from four chromosome $4 \mathrm{BAC}$ clones mapped in the vicinity of Rphq2 (Fig. 5). Microcolinearity among the regions in rice and in barley was well conserved. Nevertheless, an inversion was observed in the order of the EST-based markers WBE110 and WBE113. Many studies reported such small translocations while saturating regions of barley leaf rust resistance genes $R p h 7, r p h 16$, and $R p h 5$, respectively (Brunner et al. 2003; Mammadov et al. 2005; Perovic et al. 2004). Other reports indicated more complex deviation from microcolinearity during fine-mapping QTL for malting quality and for resistance to Fusarium head blight (Han et al. 1998; Liu et al. 2006).

Finally, the 35 molecular markers identified in the target region surrounding $R p h q 2$ were used to genotype a population of 38 subNIL and to generate a high-resolution genetic map of the region.

\section{Feasibility of cloning $R p h q 2$.}

$R p h q 2$ is located on chromosome $2 \mathrm{HL}$ in the $2 \mathrm{~L} 1.0$ region. This region was described by Dilbirligi and associates (2005) as a gene-rich region with a high level of similarity between wheat and barley. Within the $2 \mathrm{~L} 1.0$ region of barley, the region of $R p h q 2$ was described as having the highest amount of recombination events (Dilbirligi et al. 2005). Still, the amount of recombination observed in our segregating population of 923 $\mathrm{BC}_{4} \mathrm{~S}_{3}$ plants was twofold reduced compared with the amount of recombination observed earlier in the RIL population.

The LP on the 38 subNIL was estimated two times on five seedlings per line. We needed data from those two experiments to separate two significantly distinct groups for LP that unambiguously placed the locus of the gene responsible for the QTL effect between markers WBE114 and WBE115 (Fig. 6). The assessment of the effect of Rphq2 is not as straightforward as it is for major disease resistance genes that typically inherit according to Mendelian ratios. Yet, we anticipate that the amplitude of the effect of Rphq2 is large enough to pursue the map-based cloning procedure.

$R p h q 2$ was fine mapped as a single, incompletely dominant gene in a $0.11-\mathrm{cM}$ genetic interval flanked by two EST-based markers (i.e., WBE114 and WBE115). The new position of $R p h q 2$ is only $0.6 \mathrm{cM}$ distal from the position of its AFLP peak marker E38M54-294 (Figs. 4 and 6) detected in the original study of Qi and associates (1998b). This supports the idea that QTL-mapping experiments are more accurate than is generally believed (Price 2006). In rice, the homologs of WBE114 and WBE115 delimit a physical interval of 31.4 to $69.7 \mathrm{~kb}$. In barley, the megabase/centimorgan relationship in the syntenic interval is estimated at 1.1 to 1.8 (Künzel et al. 2000; Stephens et al. 2004), suggesting a physical interval of 121 to $198 \mathrm{~kb}$. 
Thus, it should be relatively easy to build a BAC contig and to close the gap between WBE114 and WBE115 using a BAC library. Two such libraries already are available in barley for cvs. Morex and Cebada Capa (Isidore et al. 2005; Yu et al. 2000). Nevertheless, to maximize the chance of physically identifying the gene underlying $R p h q 2$, we currently are constructing a new BAC library from the partially resistant cv. Vada.

\section{Identification of candidate genes}

in rice and in barley to explain Rphq2.

Knowledge of the biological role of genes underlying QTL for disease resistance remains limited and, at present, the only clue is obtained by the identification of candidate genes that coincide with the QTL. Two broad classes of genes concerned with plant defense are those involved in the recognition process and those involved in the defense-response process. It has been reported that modification of a monogenic race-specific resistance gene can give rise to a partial resistance gene $(\mathrm{Li}$ et al. 1999). After inspection of all predicted genes described in the refined $R p h q 2$ syntenic interval in rice, we did not find any homolog of such a major disease resistance gene. However, in the same syntenic target interval, genes involved in the defense-response process were identified. Six predicted rice genes on OSJNBa0039K24 encode for peroxidase proteins, three of which are homologous to WBE114. Genes of this peroxidase cluster might be candidates to explain Rphq2. Several authors already reported the association between peroxidase genes and loci for quantitative resistance (Faris et al. 1999; Marcel et al. 2007; Ramalingam et al. 2003). We also identified in the rice syntenic region another predicted gene with high sequence similarity to a mitogen-activated protein kinase kinase kinase (MAP3K). Members of the MAP3K family are crucial for early defense signaling and cellular stress response to bacterial and fungal pathogens (Asai et al. 2002). Nevertheless, the gene responsible for the Rphq2 effect in barley also might have no counterpart in rice. A wheat-rice comparative genomics analysis indicated that gene evolution occurs preferentially at the ends of chromosomes (See et al. 2006). Telomeres are hot spots for all types of recombination. As a consequence, chromosomes loose synteny from each other at a faster rate in such high-recombination regions. Moreover, rearrangements in resistance gene regions that reduce microsynteny often have prevented the straightforward identification of a candidate gene by proxy (Brueggeman et al. 2002; Brunner et al. 2003; Perovic et al. 2004).

In barley, the Rphq2 locus has been transferred to European cultivars from the botanical barley line ' $H$. laevigatum' together with the tightly linked race-specific resistance genes MlLa and $R d g l a$ for resistance to powdery mildew and to leaf stripe, respectively (Arru et al. 2002; Giese et al. 1993). In this study, MlLa mapped distally from Rphq2, ruling out the possibility that the same gene is responsible for quantitative resistance to leaf rust and qualitative resistance to powdery mildew. Arru and associates (2002) mapped $R d g l a$ approximately $10 \mathrm{cM}$ proximal from $R p h q 2$. In a leaf stripe disease test, L94-Rphq2 also was as susceptible as the susceptible parent L94 (N. Pecchioni, personal communication), excluding as well the possibility that Rphq2 and Rdgla are the same gene. In barley, approximately 16 race-specific resistance genes for leaf rust (designated as Rph loci) have been reported (Franckowiak et al. 1997). None of them mapped on the long arm of chromosome 2H (Qi et al. 1998b). Nevertheless, a major gene for resistance to leaf rust has been detected at the distal end of chromosome $2 \mathrm{HL}$ in an introgression from the bulbous barley grass $\mathrm{H}$. bulbosum (Pickering et al. 2000). It would be interesting to determine whether the $H$. bulbosum resistance to barley leaf rust is allelic to Rphq2 or not. In another study, Jafary and associates
(2006) mapped a QTL for resistance to a heterologous rust species, Puccinia persistens, at the same position as Rphq2. This suggests the interesting possibility that this QTL determines basal resistance not only to $P$. hordei, but also to one or more rust taxa to which barley is a (near) nonhost species.

\section{MATERIALS AND METHODS}

\section{Disease evaluations at the seedling stage.}

In all experiments, we used the long-time standard $P$. horde $i$ isolate 1.2.1. The inoculum preparation and the inoculation were performed as described by Marcel and associates (2007). The LP on each plant was evaluated and the RLP on seedlings (RLP50S) calculated relative to the LP on L94 seedlings, where L94 was set at 100, as described by Parlevliet (1975).

We used the avirulent $B$. graminis f. sp. hordei isolate C15, kindly provided by Dr. M. S. Hovmøller (Danish Institute of Agricultural Sciences), to map the resistance gene MlLa on our set of subNIL. The seed were sown in trays of 37 by 39 $\mathrm{cm}$. Four seeds were sown for each genotype. The first leaf of each seedling was fixed horizontally on the soil, adaxial side up, and trays were placed in a settling tower. Leaves infected with powdery mildew were collected from plants of 'Manchuria' and the spores were blown on 2-week-old seedlings by air compression within the settling tower. Each box received an average of approximately 20 spores $/ \mathrm{mm}^{2}$. After 7 days of incubation in a greenhouse compartment (temperature, 20 and $18^{\circ} \mathrm{C}$ day and night, respectively, with $16 \mathrm{~h}$ of light; relative humidity, 60\%), the ITs were scored according to the 0-to-4 scale proposed by Mains and Dietz (1930). MlLa confers an intermediate reaction type characterized by an IT of 3 . The subNIL, with an average IT $<3.5$, were considered to carry the Vada allele of MlLa whereas the subNIL with an average IT > 3.5 were considered to carry the L94 allele of MlLa.

\section{NIL development.}

NIL were developed previously by incorporating the QTL $R p h q 2$, Rphq3, and Rphq4 (Qi et al. 1998b) into an L94-susceptible genetic background (Van Berloo et al. 2001). L94$R p h q 2$ and L94-Rphq3 were crossed to select a new NIL containing both QTL in homozygous condition. To verify their quality, the L94 NIL were genotyped with an additional 11 AFLP primer combinations that resulted in 226 AFLP markers in L94 ×Vada (Marcel et al. 2007).

The reciprocal NIL were developed by introgressing the L94 allele of Rphq2, Rphq3, Rphq4, and Rphq6 in the genetic background of Vada. Twenty AFLP primer combinations were selected to screen the $\mathrm{BC}_{3}$ generation of the Vada NIL. These primer combinations resulted in 157 informative amplified fragments that occurred in L94 but not in Vada. For the next backcross generations, we used only the primer combinations amplifying AFLP markers which detected L94-derived fragments in the previous generation. Additionally, 10 microsatellites and 9 locus-specific PCR markers were used to cover areas of the genetic linkage map uncovered by the AFLP markers or to monitor the introgressions carrying the target QTL.

The RLP50S on the L94 NIL and Vada NIL was evaluated in six experiments. Each experiment contained five seedlings per genotype.

\section{Microscopic characterization of the L94 NIL.}

The reactions of L94, Vada, and NIL were characterized by fluorescence microscopy. Ten seedlings of each genotype, sown in a single tray, were inoculated with the $P$. horde $i$ isolate 1.2.1. Middle segments of 3 to $4 \mathrm{~cm}^{2}$ from the first leaves of two seedlings per genotype were collected at $110 \mathrm{~h}$ post inoculation. The eight remaining seedlings were used to measure the 
LP on each genotype. This experiment was performed two times. The collected segments were processed for fluorescence microscopy (Rohringer et al. 1977); however, instead of Calcofluor, we used Uvitex 2B for staining (Ciba-Geigy, Basel, Switzerland). The preparations were examined at $\times 100$ to $\times 400$ magnification with an epifluorescence microscope Axiophot I (Zeiss, Oberkochen, Germany). From 62 to 132 (average 98) infection units per leaf segment were scored and classified according to their stage of development (Niks 1982). Infection units that had not formed any haustorial mother cell were ignored. Infection units that formed a primary infection hypha and no more than six haustorial mother cells were considered as early aborted. Infection units with more than six haustorial mother cells were classified as established. Necrotic host cells displayed a golden yellow autofluorescence. The number of infection units associated with host cell necrosis was recorded. We measured the longest diameter of the established colonies by eye-piece micrometer.

\section{Conversion of RFLP markers to locus-specific PCR markers.}

Twenty-three primer pairs were designed on barley RFLP sequences to develop markers flanking Rphq2, Rphq3, and $R p h q 4$. The DNA sequences were downloaded from the GrainGenes database. Primer design and sequence analysis were done with the Lasergene software (DNASTAR 6.1 Inc.; Madison, WI, U.S.A.). Polymorphism detection was performed as described by Bai and associates (2004) or by direct sequencing of PCR products (BaseClear, Leiden, The Netherlands). Detailed information on the locus-specific PCR markers used in this study can be obtained from Marcel and associates (2007).

The sequences of two additional primer pairs were obtained from Hori and associates (2005) for k00345 and from Mohler and Jahoor (1996) for MWG097.

\section{Marker-assisted selection with microsatellite markers.}

The microsatellites used for the marker-assisted selection (MAS) of Rphq2 were GBM1012, GBM1475, GBMS216, and GBMS128b and for the MAS of Rphq3 were Bmac0018, Bmag0009, HVM14, HVM22a, HVM65, HVM74, GBM1063, and GBM1076. No microsatellite was found within the Rphq4 introgression. PCR conditions were according to Varshney and associates (2007). The primers were synthesized and the reverse primers labeled with near-infrared fluorescent dye at Biolegio BV (Nijmegen, The Netherlands). The PCR product was loaded on a $5.5 \%$ denaturing polyacrylamide gel $(5.5 \%$ Ready to use Gel Matrix; KB Plus, Westburg, The Netherlands) and visualized on a LI-COR 4200 DNA automated sequencer (LI-COR Biosciences, Lincoln, NE, U.S.A.).

\section{BSA with AFLP.}

A BSA was performed with AFLP primer combinations on the parents, L94 and Vada, on the NIL L94-Rphq2, and on a susceptible and a resistant pool of RILs derived from L94 $\times$ Vada. The pools were composed of equal volumes of AFLP preamplification products of seven RILs having the L94 alleles for the markers flanking $R p h q 2$ and eight RILs having the Vada alleles for the same flanking markers for the susceptible and the resistant pools, respectively. The AFLP fingerprints were generated as described by Marcel and associates (2007). The analysis was conducted exclusively with PstI/MseI-restricted DNA.

AFLP markers were converted to locus-specific PCR markers according to the method of Brugmans and associates (2003).

\section{Comparative mapping in rice and barley.}

We performed targeted synteny-based marker saturation for the Rphq2 locus following the procedure proposed by Perovic and associates (2004). The blastn function of the KOME database (Kikuchi et al. 2003) was used for the homology search between barley EST and rice clones. The predicted rice coding sequences of the identified BAC clones were used for blastn analysis in the barley TIGR Gene Indices database. Annotation and gene prediction of rice $\mathrm{BAC}$ sequences was obtained from the TIGR Rice Genome Browser web page. The barley EST with the highest homology to each predicted rice gene, above a threshold $\mathrm{E}$ value $\leq 10^{-15}$, was selected as candidate probe for genetic mapping.

\section{Substitution mapping with subNIL.}

Two $\mathrm{BC}_{4} \mathrm{~S}_{1}$ plants were identified that were heterozygous for the DNA introgressions spanning the Rphq2 region. Their $\mathrm{BC}_{4} \mathrm{~S}_{2}$ progenies were genotyped with the microsatellites GBM1475 and GBMS216 to select Vada-rphq2 and Vada$r p h q 2^{b}$ and to identify plants that recombined within the introgression. Ten $\mathrm{BC}_{4} \mathrm{~S}_{3}$ seedlings per recombinant line were tested with the flanking microsatellites to identify plants in the progenies that were homozygous for the recombined marker allele. Those plants were selfed to obtain subNIL. Therefore, subNIL are homozygous lines that have recombined within the segment introgressed in the corresponding NIL. All subNIL were genotyped with all molecular markers located distally to GBM1475 to construct a high-resolution genetic map of the $R p h q 2$ region. The subNIL were subjected to disease tests. The LP of $P$. hordei 1.2.1 was estimated in two experiments on five seedlings per subNIL. The IT against $B$. graminis $\mathrm{C} 15$ was estimated on four seedlings per subNIL.

Total DNA was extracted from leaves of the $\mathrm{BC}_{4} \mathrm{~S}_{2}$ and $\mathrm{BC}_{4} \mathrm{~S}_{3}$ plants following a method based on alkaline $(\mathrm{NaOH})$ solution described by Wang and associates (1993), adjusted for a 96-well format. This simple method allowed the extraction of DNA from a large number of plants in a limited amount of time, which suited the screening of large populations needed to identify rare recombinants.

\section{Statistical analyses.}

Linkage analyses were done with JoinMap 3.0 (Van Ooijen and Voorrips 2001) applying the Kosambi's mapping function. QTL mapping was performed using MapQTL 5.0 (Van Ooijen 2004). Interval mapping was run and markers at the log of the likelihood ratio peaks were taken as cofactors for running the restricted-MQM mapping method (rMQM). The proportion of explained phenotypic variance was estimated with the rMQM results.

The analyses of variance with RLP on NIL and subNIL and percentage of early abortion and colony length on NIL were performed with GenStat Release 8.1 (VSN International Ltd., Hemel Hempstead, U.K.). A Duncan's multiple range test $(P<$ $0.05)$ was used to compare all pairs of means between NIL. The least significant difference $(P<0.05)$ was used to declare the mean of a subNIL significantly different or not from the mean of Vada.

\section{ACKNOWLEDGMENTS}

This project was sponsored by the Dutch Organization for Scientific Research (NWO, project number 809.36.001) and is being supported by the European Commission through the sixth framework program's integrated project, BIOEXPLOIT (contract number FOOD-CT-2005-513959). We thank M. S. Hovmøller of the Danish Institute of Agricultural Sciences for providing the powdery mildew isolate $\mathrm{C} 15$ carrying avirulence to $M l L a$; N. Stein and M. Röder of the Institute of Plant Genetics and Crop Plant Research (IPK) for providing primer sequences of GBM- and GBMS-microsatellite markers; B. Gorguet, M. T. Ta, and G. R. Rodenas for assistance in developing the near-isogenic lines; and A. Vels for technical assistance in the greenhouse activities. 


\section{LITERATURE CITED}

Alonso-Blanco, C., and Koornneef, M. 2000. Naturally occurring variation in Arabidopsis: An underexploited resource for plant genetics. Trends Plant Sci. 5:22-29.

Arru, L., Niks, R. E., Lindhout, P., Valé, G., Francia, E., and Pecchioni, N. 2002. Genomic regions determining resistance to leaf stripe (Pyrenophora graminea) in barley. Genome 45:460-466.

Asai, T., Tena, G., Plotnikova, J., Willmann, M. R., Chiu, W.-L., GomezGomez, L., Boller, T., Ausubel, F. M., and Sheen, J. 2002. MAP kinase signalling cascade in Arabidopsis innate immunity. Nature 415:977-983.

Bai, Y., Feng, X., van der Hulst, R., and Lindhout, P. 2004. A set of simple PCR markers converted from sequence specific RFLP markers on tomato chromosomes 9 to 12. Mol. Breed. 13:281-287.

Brueggeman, R., Rostoks, N., Kudrna, D., Kilian, A., Han, F., Chen, J., Druka, A., Steffenson, B., and Kleinhofs, A. 2002. The barley stem rust-resistance gene Rpgl is a novel disease-resistance gene with homology to receptor kinases. Proc. Natl. Acad. Sci. U.S.A. 99:93289333.

Brugmans, B., van der Hulst, R., Visser, R. G. F., Lindhout, P., and van Eck, H. J. 2003. A new and versatile method for the successful conversion of AFLP markers into simple single locus markers. Nucleic Acids Res. 31:1-9.

Brunner, S., Keller, B., and Feuillet, C. 2003. A large rearrangement involving genes and low-copy DNA interrupts the microcollinearity between rice and barley at the Rph7 locus. Genetics 164:673-683.

Devos, G. 2005. Updating the 'Crop Circle'. Curr. Opin. Plant Biol. 8:155162.

Dilbirligi, M., Erayman, M., and Gill, K. S. 2005. Analysis of recombination and gene distribution in the $2 \mathrm{~L} 1.0$ region of wheat (Triticum aestivum L.) and barley (Hordeum vulgare L.). Genomics 86:47-54.

Dros, J. 1957. The creation and maintenance of two spring barley varieties. Euphytica 6:45-48.

Faris, J. D., Li, W. L., Liu, D. J., Chen, P. D., and Gill, B. S. 1999. Candidate gene analysis of quantitative disease resistance in wheat. Theor. Appl. Genet. 98:219-225.

Franckowiak, J. D., Jin, Y., and Steffenson, B. J. 1997. Recommended allele symbols for leaf rust resistance genes in barley. Barley Genet. News1. 27:36-44

Giese, H., Holm-Jensen, A. G., Jensen, H. P., and Jensen, J. 1993. Localization of the Laevigatum powdery mildew resistance gene to barley chromosome 2 by the use of RFLP markers. Theor. Appl. Genet. 85:897-900

Graner, A., Jahoor, A., Schondelmaier, J., Siedler, H., Pollen, K., Fischbeck, G., Wenzel, G., and Herrmann, R. G. 1991. Construction of an RFLP map of barley. Theor. Appl. Genet. 83:250-256.

Han, F., Kleinhofs, A., Ullrich, S. E., Kilian, A., Yano, M., and Sasaki, T. 1998. Synteny with rice: Analysis of barley malting quality QTLs and rpg4 chromosome regions. Genome 41:373-380.

Hori, K., Sato, K., Nankaku, N., and Takeda, K. 2005. QTL analysis in recombinant chromosome substitution lines derived from a cross between Hordeum vulgare ssp. vulgare and Hordeum vulgare ssp. spontaneum. Mol. Breed. 16:295-311.

Isidore, E., Scherrer, B., Bellec, A., Budin, K., Faivre-Rampant, P., Waugh, R., Keller, B., Caboche, M., Feuillet, C., and Chalhoub, B. 2005. Direct targeting and rapid isolation of BAC clones spanning a defined chromosome region. Funct. Integr. Genomics 5:97-103.

Jafary, H., Szabo, L. J., and Niks, R. E. 2006. Innate nonhost immunity in barley to different heterologous rust fungi is controlled by sets of resistance genes with different and overlapping specificities. Mol. Plant-Microbe Interact. 19:1270-1279.

Jander, G., Norris, S. R., Rounsley, S. D., Bush, D. F., Levin, I. M., and Last, R. L. 2002. Arabidopsis map-based cloning in the post-genome era. Plant Physiol. 129:440-450.

Jensen, H. P., and Jørgensen, J. H. 1992. Genetics of 'Laevigatum' resistance and virulence in barley and powdery mildew. Barley Genet. Newsl. 21:34-37.

Karakousis, A., Gustafson, J. P., Chalmers, K. J., Barr, A. R., and Langridge, P. 2003. A consensus map of barley integrating SSR, RFLP, and AFLP markers. Aust. J. Agric. Res. 54:1173-1185.

Kikuchi, S., Satoh, K., Nagata, T., Kawagashira, N., Doi, K., Kishimoto, N., Yazaki, J., Ishikawa, M., Yamada, H., Ooka, H., Hotta, I., Kojima, K., Namiki, T., Ohneda, E., Yahagi, W., Suzuki, K., Li, C. J., Ohtsuki, K., Shishiki, T., Otomo, Y., Murakami, K., Iida, Y., Sugano, S., Fujimura, T., Suzuki, Y., Tsunoda, Y., Kurosaki, T., Kodama, T., Masuda, H., Kobayashi, M., Xie, Q., Lu, M., Narikawa, R., Sugiyama, A., Mizuno, K., Yokomizo, S., Niikura, J., Ikeda, R., Ishibiki, J., Kawamata, M., Yoshimura, A., Miura, J., Kusumegi, T., Oka, M., Ryu, R., Ueda, M., Matsubara, K., Kawai, J., Carninci, P., Adachi, J., Aizawa, K., Arakawa, T., Fukuda, S., Hara, A., Hashidume, W., Hayatsu, N., Imotani, K., Ishii,
Y., Itoh, M., Kagawa, I., Kondo, S., Konno, H., Miyazaki, A., Osato, N., Ota, Y., Saito, R., Sasaki, D., Sato, K., Shibata, K., Shinagawa, A., Shiraki, T., Yoshino, M., and Hayashizaki, Y. 2003. Collection, mapping, and annotation of over 28,000 cDNA clones from japonica rice. Science 301:376-379.

Kleinhofs, A., Kilian, A., Saghai Maroof, M. A., Biyashev, R. M., Hayes, P., Chen, F. Q., Lapitan, N., Fenwick, A., Blake, T. K., Kanazin, V., Ananiev, E., Dahleen, L., Kudrna, D., Bollinger, J., Knapp, S. J., Liu, B., Sorrells, M., Heun, M., Franckowiak, J. D., Hoffman, D., Skadsen, R., and Steffenson, B. J. 1993. A molecular, isozyme and morphological map of the barley (Hordeum vulgare) genome. Theor. Appl. Genet. 86:705-712.

Kraakman, A. T. W., Martínez, F., Mussiraliev, B., van Eeuwijk, F. A., and Niks, R. E.. 2006. Linkage disequilibrium mapping of morphological, resistance, and other agronomically relevant traits in modern spring barley cultivars. Mol. Breed. 17:41-58.

Künzel, G., Korzun, L., and Meister, A. 2000. Cytologically integrated physical restriction fragment length polymorphism maps for the barley genome based on translocation breakpoints. Genetics 154:397-412.

Langridge, P., Karakousis, A., Collins, N., Kretschmer, J., and Manning, S. 1995. A consensus linkage map of barley. Mol. Breed. 1:389-395.

Li, Z.-K., Luo, L. J., Mei, H. W., Paterson, A. H., Zhao, X. H., Zhong, D. B., Wang, Y. P., Yu, X. Q., Zhu, L., Tabien, R., Stansel, J. W., and Ying, C. S. 1999. A "defeated" rice resistance gene acts as a QTL against a virulent strain of Xanthomonas oryzae pv. oryzae. Mol. Gen. Genet. 261:58-63.

Liu, S., Zhang, X., Pumphrey, M. O., Stack, R. W., Gill, B. S., and Anderson, J. A. 2006. Complex microcolinearity among wheat, rice, and barley revealed by fine mapping of the genomic region harboring a major QTL for resistance to Fusarium head blight in wheat. Funct. Integr. Genomics 6:83-89.

Mains, E. B., and Dietz, S. M. 1930. Physiologic forms of barley mildew, Erysiphe graminis hordei Marchal. Phytopathology 20:229-239.

Mammadov, J. A., Steffenson, B. J., and Saghai Maroof, M. A. 2005. High-resolution mapping of the barley leaf rust resistance gene Rph5 using barley expressed sequence tags (ESTs) and synteny with rice. Theor. Appl. Genet. 111:1651-1660.

Marcel, T. C., Varshney, R. K., Barbieri, M., Jafary, H., de Kock, M. J. D., Graner, A., and Niks, R. E. 2007. A high-density consensus map of barley to compare the distribution of QTLs for partial resistance to Puccinia hordei and of defence gene homologues. Theor. Appl. Genet. 114:487-500.

Mohler, V., and Jahoor, A. 1996. Allele-specific amplification of polymorphic sites for the detection of powdery mildew resistance loci in cereals. Theor. Appl. Genet. 93:1078-1082.

Moore, G., Devos, K. M., Wang, Z., and Gale, M. D. 1995. Grasses, line up and form a circle. Curr. Biol. 5:737-739.

Neervoort, W. J., and Parlevliet, J. E. 1978. Partial resistance of barley to leaf rust, Puccinia hordei. V. Analysis of the components of partial resistance in eight barley cultivars. Euphytica 27:33-39.

Niks, R. E. 1982. Early abortion of colonies of leaf rust, Puccinia hordei, in partially resistant barley seedlings. Can. J. Bot. 60:714-723.

Niks, R. E. 1983. Haustorium formation by Puccinia hordei in leaves of hypersensitive, partially resistant, and nonhost plant genotypes. Phytopathology 73:64-66.

Niks, R. E. 1986. Failure of haustorial development as a factor in slow growth and development of Puccinia hordei in partially resistant barley seedlings. Physiol. Mol. Plant P. 28:309-322.

Niks, R. E., Habekuß, A., Bekele, B., and Ordon, F. 2004. A novel major gene on chromosome $6 \mathrm{H}$ for resistance of barley against the barley yellow dwarf virus. Theor. Appl. Genet. 109:1536-1543.

Parlevliet, J. E. 1975. Partial resistance of barley to leaf rust, Puccinia hordei. I. effect of cultivar and development stage on latent period. Euphytica 24:21-27.

Paterson, A. H., DeVerna, J. W., Lanini, B., and Tanksley, S. D. 1990. Fine Mapping of quantitative trait loci using selected overlapping recombinant chromosomes, in an interspecies cross of tomato. Genetics 124:735-742.

Perovic, D., Stein, N., Zhang, H., Drescher, A., Prasad, M., Kota, R., Kopahnke, D., and Graner, A. 2004. An integrated approach for comparative mapping in rice and barley with special reference to the Rph16 resistance locus. Funct. Integr. Genomics 4:74-83.

Pickering, R. A., Malyshev, S., Künzel, G., Johnston, P. A., Korzun, V., Menke, M., and Schubert, I. 2000. Locating introgressions of Hordeum bulbosum chromatin within the $H$. vulgare genome. Theor. Appl. Genet. 100:27-31.

Price, A. H. 2006. Believe it or not, QTLs are accurate! Trends Plant Sci. 11:213-216.

Qi, X., Stam, P., and Lindhout, P. 1996. Comparison and integration of four barley genetic maps. Genome 39:379-394. 
Qi, X., Stam, P., and Lindhout, P. 1998a. Use of locus-specific AFLP markers to construct a high-density molecular map in barley. Theor. Appl. Genet. 96:376-384.

Qi, X., Niks, R.E., Stam, P., and Lindhout, P. 1998b. Identification of QTLs for partial resistance to leaf rust (Puccinia hordei) in barley. Theor. Appl. Genet. 96:1205-1215.

Ramalingam, J., Vera Cruz, C. M., Kukreja, K., Chittoor, J. M., Wu, J.-L., Lee, S. W., Baraoidan, M., George, M. L., Cohen, M. B., Hulbert, S. H., Leach, J. E., and Leung, H. 2003. Candidate defense genes from rice, barley, and maize and their association with qualitative and quantitative resistance in rice. Mol. Plant-Microbe Interact. 16:14-24.

Remington, D. L., Ungerer, M. C., and Purugganan, M. D. 2001. Mapbased cloning of quantitative trait loci: Progress and prospects. Genet. Res. 78:213-218.

Rohringer, R., Kim, W. K., Samborski, D. J., and Howes, N. K. 1977. Calcofluor: An optical brightener for fluorescence microscopy of fungal plant parasites in leaves. Phytopathology 67:808-810.

Salvi, S., and Tuberosa, R. 2005. To clone or not to clone plant QTLs: Present and future challenges. Trends Plant Sci. 10:298-304.

Schmierer, D., Drader, T., Horsley, R., and Kleinhofs, A. 2003. Saturation mapping of a major Fusarium head blight QTL on barley chromosome 2H. Pages 36-39 in: National Fusarium Head Blight Forum Proceeding. S. M. Canty, J. Lewis, and R. W. Ward, eds. Michigan State University, East Lansing, MI, U.S.A.

See, D. R., Brooks, S., Nelson, J. C., Brown-Guedira, G., Friebe, B., and Gill, B. S. 2006. Gene evolution at the ends of wheat chromosomes. Proc. Natl. Acad. Sci. U.S.A. 103:4162-4167.

Stephens, J. L., Brown, S. E., Lapitan, N. L. V., and Knudson, D. L. 2004 Physical mapping of barley genes using an ultrasensitive fluorescence in situ hybridization technique. Genome 47:179-189.

Thomsen, S. B., Jensen, H. P., Jensen, J., Skou, J. P., and Jørgensen, J. H. 1997. Localization of a resistance gene and identification of sources of resistance to barley leaf stripe. Plant Breed. 116:455-459.
Van Berloo, R., Aalbers, H., Werkman, A., and Niks, R. E. 2001. Resistance QTL confirmed through development of QTL-NILs for barley leaf rust resistance. Mol. Breed. 8:187-195.

Van Ooijen, J. W. 2004. MapQTL 5, Software for the Mapping of Quantitative Trait Loci in Experimental Populations. Kyazma B.V., Wageningen, The Netherlands.

Van Ooijen, J. W., and Voorrips, R. E. 2001. JoinMap Version 3.0, Software for the Calculation of Genetic Linkage Maps. Plant Research International, Wageningen, The Netherlands.

Varshney, R. K., Marcel, T. C., Ramsay, L., Russell, J., Röder, M., Stein, N., Waugh, R., Langridge, P., Niks, R. E., and Graner, A. 2007. A high density barley microsatellite consensus map with 775 SSR loci. Theor. Appl. Genet. 114:1091-1103.

Wang, H., Qi, M., and Cutler, A. J. 1993. A simple method of preparing plant samples for PCR. Nucleic Acid Res. 21:4153-4154.

$\mathrm{Xu}$, Y., McCouch, S. R., and Zhang, Q. 2005. How can we use genomics to improve cereals with rice as a reference genome? Plant Mol. Biol. 59:7-26.

Yu, Y., Tomkins, J. P., Waugh, R., Frisch, D. A., Kudrna, D., Kleinhofs, A., Brueggeman, R. S., Muehlbauer, G. J., Wise, R. P., and Wing, R. A. 2000. A bacterial artificial chromosome library for barley (Hordeum vulgare L.) and the identification of clones containing putative resistance genes. Theor. Appl. Genet. 101:1093-1099.

\section{AUTHOR-RECOMMENDED INTERNET RESOURCES}

GrainGenes database: wheat.pw.usda.gov/GG2/index.shtml

The Institute for Genomic Research (TIGR) rice genome browser: www.tigr.org/tigr-scripts/osa1_web/gbrowse/rice

Knowledge-based Oryza molecular biological encyclopedia (KOME): cdna01.dna.affrc.go.jp/cDNA

TIGR Gene Indices: compbio.dfci.harvard.edu/tgi/tgipage.html 